www.jmscr.igmpublication.org

Index Copernicus Value: 79.54

ISSN (e)-2347-176x ISSN (p) 2455-0450

crossrefDOI: https://dx.doi.org/10.18535/jmscr/v7i1.194

\title{
A questionnaire based study to assess insulin injection practices and hypoglycemic awareness among diabetic patients in a tertiary care centre
}

\author{
Authors \\ Viral Sangwan, Birender Singh, Roopa Malik, Manjari Garg \\ PT BDS PGIMS Rohtak \\ Email: drviralsangwan@yahoo.com, Mobile: 9896130304
}

\begin{abstract}
Introduction: Effective insulin injection technique is essential to ensure effective management of diabetes. The purpose of this study is to assess the current situation of insulin injection techniques in patients with diabetes.

Material and Methods: It was a cross sectional study conducted on a total of 200 patients of type 1 and type 2 Diabetes who were using insulin injections for last three months. They were accessed regarding knowledge and practice of insulin injection techniques and hypoglycaemic management using a questionnaire. Observations regarding injection site problems were made.

Results: Mean age of patients was $34.68 \pm 17.43$ years. $97.5 \%$ had formal education regarding injection technique. 51.5\% subjects were using pen devices for insulin administration. $83.5 \%$ did not know about priming. $84 \%$, preferred abdomen for injection.

$89.5 \%$ were changing site regularly before injection. $76 \%$ were using 90 degree angle for injection. $83.5 \%$ subjects used to make fold of skin before injection. $43 \%$ were releasing skin immediately after injecting. $72.5 \%$ subjects did not clean site before injection. $64 \%$ never allowed injection to reach room temperature. $97 \%$ did not understand sick day rule.

$51 \%$ subjects used to have bruising and bleeding. 53.5\% noticed fluid leakage after injection. $42 \%$ used to rub their site after injection. $87.5 \%$ had glucometer. 25\% used to check their blood glucose 3 to 4 times in a day. Out of 200, 72\% did not know about the symptoms and signs of hypoglycaemia.

Conclusion: There is significant gap between recommended injection technique and current injection practice among Indian diabetics.

Keywords: Diabetes, Insulin, Questionaire.
\end{abstract}

\section{Introduction}

Diabetes is continuously gaining the status of potential epidemic in world. Current report estimates that there are 415 millions of people with diabetes in the world. ${ }^{1}$ India has estimated prevalence rate of about $7.8 \%$ and ranked second in world with approximately 69.2 million diabetic patients. ${ }^{1}$ Statistics from developed countries shows more than $30 \%$ of all diabetics use insulin singly or in combination with other though this figure may be lower for India. ${ }^{2}$

Effective insulin injection technique is essential to ensure effective management of diabetes. Those who administer insulin should understand how it works, in particular its link to blood glucose levels. Potential injection sites include the 
abdomen, thigh, buttock and arm. Insulin can be injected either using a prefilled insulin pen or insulin syringe and vial. Insulin should be stored at temperature between 2 to 8 degrees in refrigerator. $^{3}$ It should be kept at room temperature before use as cold insulin can be uncomfortable and delay absorption. ${ }^{4}$ The pen should be primed first, usually two unit to clear any air bubble to ensure accurate dosing of insulin. ${ }^{5}$ An ideal injection technique includes proper needle length, angle of insertion, skin lift and post injection hold. Skin should be raised to avoid injection into muscle. The needle should be inserted subcutaneously at 90 degree and should be held in skin for approximately 10 seconds to ensure full delivery of insulin in subcutaneous space. Incorrect injection technique can cause various local site reactions such as lipoatrophy/ lipodystrophy, lipohypertrophy, bruising and bleeding and amyloidosis.

The efficacy of injection therapy in diabetes depends on correct injection technique. To provide patients with guidance in this area and help patients inject themselves correctly, we must understand how they are currently injecting. Therefore, the purpose of this study is to assess the current situation of insulin injection techniques in patients with diabetes. There are several Indian studies with emphasis on diabetes epidemiology ${ }^{6}$, 7 but one related to knowledge; attitude and practice concerning insulin use in diabetics are limited. ${ }^{8-10}$

\section{Material and Methods}

Present study was a cross sectional study conducted on a total of 200 patients of type 1 and type 2 Diabetes Mellitus who were using insulin injections for last three months coming to Medicine and Endocrine outpatient department. They were accessed regarding knowledge and practice of insulin injection techniques and hypoglycaemic management using a questionnaire.

Newly diagnosed Diabetes Mellitus patients, patients not able to understand English or Hindi and visually impaired patients were excluded from study.

The study accessed following parameters regarding insulin injections; choice of injection site, rotation of injection site, rolling of cloudy insulin vials/ pens before use, skin fold technique, knowledge of length of needle used, needle entry angle, disinfection of site prior to injection, injection storage at home and while travelling and blood glucose monitoring.

Observations regarding injection site problems such as insulin leakage, bleeding, bruising and lipohypertrophy were made. The data on lipohypertrophy was obtained through visual inspection and palpation. Patients were asked about their knowledge of hypoglycaemic symptoms and their home management.

Statistical analysis was done using SPSS version 20 (IBM SPSS Statistics Inc., Chicago, Illinois, USA) Windows software program. The variables were assessed for normality using the Kolmogorov Smirnov test. Descriptive statistics included computation of percentages, means and standard deviations.

\section{Results}

The mean age of patients was $34.68 \pm 17.43$ years. Highest number of subjects, $30.5 \%$ was found in 15-30 years age group followed by $27.5 \%$ in 30 45 years and $19 \%$ in $45-60$ years. $97.5 \%$ subjects had received a formal education regarding injection technique and $71 \%$ wanted to receive more education regarding injection technique. $51.5 \%$ subjects were using pen devices for insulin administration. $93.5 \%$ and $88 \%$ subjects were unaware of different quantity of insulin vials and orange colored insulin syringes respectively. 83.5\% subjects did not know about priming. 95\% subjects did not have knowledge about length of needle and 166 subjects, $83 \%$ were using needle irregularly usually more than three times and the reason for reuse was lack of knowledge in 99, by their convenience in 29 and to save money in 17 subjects. 84\% (168) subjects, preferred abdomen for injection as a single or in combination of other 
sites followed by arm in $10.5 \%$ (21), thigh in $4 \%$ (8) and buttocks in $1.5 \%$ (3).

Only $25(12.5 \%)$ subjects agreed with regular inspection of injection site by health professional while 149 (74.5\%) did not remember it. Most of the subjects 179 ( $89.5 \%$ ) were changing their site regularly before injection. 152 subjects, $76 \%$ were using 90 degree angle for injection. 167 (83.5\%) subjects used to make fold of skin before injection. 86 subjects, $43 \%$ were releasing skin immediately after injecting followed by 67 (33.5\%) less than 10 seconds and $45(22.5 \%)$ more than 10 seconds. 155 subjects $(72.5 \%)$ subjects did not clean site before injection. 33 subjects, $16.5 \%$ were injecting insulin immediately after application of spirit. 128 subjects, 64\% never allowed injection to reach room temperature. 187 subjects, $93.5 \%$ did not inject injection through clothes. 194 subjects, 97\% did not understand sick day rule. 58 subjects, $29 \%$ were inspecting their injection site for swelling or lump regularly while $66,33 \%$ subjects did not inspect.

$183,91.5 \%$ subjects did not inject insulin at swelling or lump site and small number of subjects $17,8.5 \%$ did it. $35,17.5 \%$ subjects used to rotate vial or pen before injection. 145 subjects, $72.5 \%$ had pain during insulin injection. $102,51 \%$ subjects used to have bruising and bleeding at injection site and 98, 49\% did not notice it. 107, $53.5 \%$ used to notice fluid leakage after injection. $42 \%$ used to rub their site after injection.175, $87.5 \%$, have glucometer.50 subjects, $25 \%$ used to check their blood glucose 3 to 4 times in a day. Out of 200, 114 subjects, $72 \%$ did not know about the symptoms and signs of hypoglycaemia. 71 subjects, $35.5 \%$ were using sugar followed by 22 , $11 \%$ coffee and $13,6.5 \%$ oral glucose if they were experiencing hypoglycaemia. 159 subjects, $79.5 \%$ never used to recheck their blood glucose after managing hypoglycaemia. 181, 90.5\% subjects never used to remove needle from their pen after each use. 196, 98\% were storing their pens and vials in refrigerator, of these $94 \%$ were storing in side compartment in refrigerator.190, $90.5 \%$ were discarding their needle and syringes into garbage. $139,69.5 \%$ were discarding without recapping. $116,58 \%$ were using ice packs while travelling for storing their injections and 20,10\% used to pack it with clothes while $33,16.5 \%$ did not take any precaution.

72 subjects, $36 \%$ used to have meal after 5 minutes of injection followed by $52,26 \%$ at 30 minutes interval and $43,22.5 \%$ after 15 minutes irrespective of type of insulin.

\section{Discussion}

The present questionnaire based study was carried out in 200 patients of diabetes mellitus who were using insulin for last 3months. Highest number of patients was found in age group 15-30 years $30.5 \%$ followed by $27.5 \%$ in $30-45$ years and $19 \%$ in 45-60 age group. About $97.5 \%$ said that they had received formal education regarding insulin injection technique. $71 \%$ wanted more education regarding injection techniques.

Most of subjects in our study were using insulin pen devices $(51.5 \%)$ followed (34\%) syringe and needle. $9.5 \%$ patients were using both pen and syring, needle. Similar to other studies pen devices were preferable to vials because they were more convenient to carry, less painful, less leakage from injection site. ${ }^{11-13}$

It is recommended that insulin pen should be primed prior to insulin administration in order to ensure free and unobstructed flow. ${ }^{14}$ Most of patients did not prime their pen devices similar to another study. ${ }^{11}$ The studies in western countries showed more than $70 \%$ were priming their pens. ${ }^{11}$ It may be due to lack of education. In our study three fourth of premix user patients were rolling their pens to mix insulin. Similarly in another study where three fifth of premix users used to mix it prior to administration and there was lower number of patients who correctly mix it. ${ }^{11}$ Lack of mixing can alter clinical response and also increase insulin requirement. ${ }^{11}$

Most of our patients were using either $8 \mathrm{~mm}$ or 4 $\mathrm{mm}$ needle. It is comparable to other studies where also the maximum number of patients were using 
$8 \mathrm{~mm}$ or less than $8 \mathrm{~mm}$ needles. ${ }^{11-13}$ Previously, it was thought that shorter needle were unable to penetrate deep enough to deliver insulin. But recent studies have demonstrated that they are indeed effective in delivering insulin, longer needle may penetrate too deeply leading to inconsistent insulin absorption and inadequate glycaemic control. ${ }^{15}$

In our study $83 \%$ of patients were using needle three or more than three times. When they were asked for reason for reusing then around $45.5 \%$ subjects said that they were never told about changing of needle after using three times. Rest were doing it for their convenience and saving money. It has recommended that needle should not be reused. ${ }^{15} \mathrm{~A}$ national survey done earlier also showed that $92.5 \%$ persons were reusing needles at least 2 times to more than 10 times. ${ }^{16}$ Another study revealed that Indian diabetic patients were injecting insulin more than 6 times with same needle. ${ }^{17}$ It can be reduced by educating people regarding harms of needle reuse e.g. loss of sterility, lubrication or damage of needle tip by bending and breakage. $^{18}$ It also raises development of lipohypertrophy. ${ }^{18}$ Making needle cheaper or free availability may solve the problem.

The anterior abdomen remained the most common site for injection in all studies. ${ }^{11-13}$ Similarly in our study anterior abdomen is most preferred site (66\%) followed by arm (11.5\%), thigh(4\%), buttock (1.5\%). Rest $18 \%$ were using anterior abdomen as a preferred site with combination of other sites. Abdomen was most common site due to ease of injection and consistency of insulin absorption. The practice of rotation in our study was comparable to international standards. It is recommended that injection site should be $3 \mathrm{~cm}$ apart. However, when we talk about the inspection of injection site for lump or swelling, only 58\% were inspecting their injection site for lump and swelling always or sometimes. Only11\% said that their injection site being regularly checked by their physician. $75 \%$ were denying that their injection sites ever checked by their treating physician.8.5\% were still injecting insulin in swelling. It is associated with inconsistent and decreased insulin absorbtion. ${ }^{19}$

Insulin delivery is an important aspect of a successful insulin therapy. Ideal injection technique includes proper needle length, rolling of pens, angle of insertion, skin lift and post injection holding of skin. The pen devices or vials should be rotated systemically at least 10 times (preferably 20 times) before injection. ${ }^{20,21}$ Well designated studies have shown that anything less risks leaving the crystals of suspension, with the attendant danger of injecting either less or more insulin than desired and varying the concentration injected. ${ }^{22}$ In our study around $76 \%$ used to inject at 90 degree. In other studies $78 \%$ were injecting at 90 degree. $^{11}$ Around $83.5 \%$ used to make skin fold for injection regularly comparable to other studies $^{12,13}$ where more than $60 \%$ were making skin fold. But $43 \%$ out of them used to release skin fold immediately after injection. This is followed by $33.5 \%$ and $22.5 \%$ who used to release their skin fold less than and more than 10 seconds respectively. In other studies about 50\% were releasing fold immediately. ${ }^{11}$ Releasing skin fold earlier increases the risk of insulin leakage from injection site and pushing needle in muscle. So, patients should be taught to count for 10 seconds after button of device has been fully depressed and then remove the needle from skin. They should also be taught that skin fold should be made by using thumb and index finger not by whole hand. The latter technique risks lifting of entire muscle, therefore promoting intramuscular injection. ${ }^{51}$ Holding for more than 10 seconds also allows full evacuation of injection in skin and better dispersion into the subcutaneous tissue. If leakage is present then waiting time should be extended. In our study $50.5 \%$ used to notice fluid leakage after injection. In other studies about $40 \%$ used to notice fluid leakage. ${ }^{11,12}$ Beside fluid leakage improper injection technique can also results in various other complications bleeding, lipohypertrophy. In our study $45.5 \%$ used to notice bleeding and bruising at injection site. 
Similarly in other studies more than $40 \%$ used to have bleeding and bruising. ${ }^{11} 22.5 \%$ were disinfecting their site with sprit before injection. Similarly in another Indian study showed only $27 \%$ were disinfecting their site prior to injection. $^{12}$ The study conducted in Nepal also showed that majority of participants did not clean their site before injection. ${ }^{13} 42 \%$ in our study massaged their injection site after injection delivery. In another study about $20.9 \%$ used to massage their injection site after administration of insulin. $^{13}$

Guidelines from western countries clearly recommend self-monitoring of blood glucose in insulin users. $^{12}$ In our study $87.5 \%$ had glucometer. $24.5 \%$ used to monitoring blood glucose 3 to 4 times, $21 \%$ used to do it 1 to 2 times. $24 \%$ were monitoring periodically in a week. Another Indian study also showed that $77.3 \%$ did get their blood glucose checked periodically.56\% knew the symptoms of hypoglycaemia but only $13 \%$ were using glucose if they were feeling symptoms of hypoglycaemia. $35.5 \%$ were using sugar.79.5\% were not rechecking their blood glucose after managing hypoglycaemia. Another survey done in India also showed that despite knowing symptoms of hypoglycaemia only a quarter of patients $(27.3 \%)$ used to carry simple sugar with them. ${ }^{12}$ As this is a lifesaving practice, this aspect need emphasis in diabetic clinic. All patients should be carefully counselled and reiterated at each follow up visit.

93\% were discarding their needles into garbage and $69.5 \%$ were discarding without recapping. Only 7\% were using sharp container for disposal. Similarly in another study almost all patients used to dispose their used needles improperly. ${ }^{13}$

Insulin should be stored in cool and dry places. Insulin pens and vials not in use should be refrigerated. Cold injection may cause local irritation to skin. So vials and pen with cartridge should be kept at room temperature for at least 30 minute before use. Pens should not be stored with needle on because air may clog needle and affect dosage delivery. In our study about $98 \%$ were storing their injections in refrigerator and 94\% were using side compartment for storage. More than $90 \%$ were not removing needle from their insulin pens in use (insulin cartridge inside).It leads to accumulation of air in pen which inversely affect insulin delivery at its predefined time.58\% were using ice packs for carrying insulin while travelling.

$36 \%$ patients used to have their meal after 5 minutes followed by $26 \%$ who had their meal after 30 minutes irrespective of type of insulin used. The guideline recommends interval of 30 minutes when injecting regular insulin and 5 minutes when injecting Lispro. There are studies which have shown no change in HBA1C level with variation of injection meal gap. ${ }^{23}$

Our study had few limitations. It was performed on single centre so it might not sufficiently generalize insulin injection practice all over India. Moreover, because of limited budget, ultrasonography and photography were not used for lipohypertrophy.

\section{Conclusion}

There is significant gap between recommended injection technique and current injection practice among Indian diabetics. Physicians/nursing staff should educate each patient properly regarding every aspect of injection technique of Insulin and disadvantages of faulty technique.

\section{References}

1. International Diabetes Federation. The International Federation (IDF) Diabetes Atlas, Seventh Edition. 2015. Available at https://www.idf.org/membership/sea/india.

2. United States Centres for Disease Control and Prevention. Diabetes data and trends: Age-adjusted percentage of adults with diabetes using diabetes medication, by type of medication, United States, 19972011 [document on the internet] 2013. Available from: http://www.cdc.gov/diabetes/statistics/med use/fig2.htm . 
3. Fit UK (2016) fit UK forum for injection technique UK: the UK injection technique recommendations. Fourth edition www.fit4diabetes.com/files

/4514/7946/3482/fit_UK_recommendation s_4th_edition.pdf

4. Hatchett $R$ The medicines refrigerator and the importance of the cold chain in the safe storage of medicines. Nurs Stand. 2017; 32: 53-63.

5. Curtis T, Moutter S. How to administer an insulin injection. Nurs stand. 2017; 32: 4146.

6. Madhu SV, Rao PV. Epidemiology of diabetes mellitus in India. In: Tripathi BB, Chandalia HB, editors. RSSDI Textbook of Diabetes Mellitus. 2nd ed. Hyderabad: Research Society for study of diabetes in India; 2008: 209-26.

7. Ramachandran A, Snehalatha C, Kapur A, Vijay V, Mohan V, Das AK, et al. High prevalence of diabetes and impaired glucose tolerance in India: National Urban Diabetes Survey. Diabetologia. 2001; 44:1094-101.

8. Shah VN, Kamdar PK, Shah N. Assessing the knowledge, attitudes and practice of type 2 diabetes among patients of Saurashtra region, Gujarat. Int J Diabetes Dev Ctries. 2009; 29:118-22.

9. Priyanka Raj CK, Angadi MM. Hospitalbased KAP study on diabetes in Bijapur, Karnataka. Indian J Med Spec. 2010; 1: 80-3.

10. Malathy R, Narmadha M, Ramesh S, Alvin JM, Dinesh BN. Effect of a diabetes counselling programme on knowledge, attitude and practice among diabetic patients in Erode district of South India. J Young Pharm. 2011; 3: 65-72.

11. Berard RL, Cameron B. Injection technique practices in a population of Canadians with diabetes: results from a recent patient/diabetes educator survey. Can J Diabetes 2015; 39: 146-51.
12. Frid AH, Hirsch LJ, Menchior AR, Morel DR, Strauss KW. Worldwide injection technique questionnaire study: population parameters and injection practices. Mayo Clin Proc. 2016; 91:1212-23.

13. Poudel R S, Srestha S, Piryani RM, Basyal B, Kaucha K, Adhikari S. Assessment of insulin injection practice among diabetes patients in a tertiary healthcare in Nepal: A preliminary study. Journal of Diabetes Research 2017; Available https://doi.org/10.1155/2017/8648316.

14. Standards of medical care in diabetes 2013. American Diabetes Association Diabetic care. 2013; 36, S11-S66.

15. Frid A, Hirsch L, Gaspar R, Hicks D, Kreugel G, Liersch J, et al. New injection recommendations for patients with diabetes. Diabetes Metab. 2010; 36: S318.

16. Kalra S, Mithal A, Sahay R, John M, Unnikrishnan AG, Saboo B et al. Indian injection technique study: population characteristics and injection practices. Diabetes Ther 2017; 8: 637-657.

17. Patil M, Sahoo J, Kamalanathan S, Selviambigapathy J, Balachandran $\mathrm{K}$, Kumar R et al. Assessment of insulin injection techniques among diabetes patients in a tertiary care centre. Diabetes Metab Syndr. 2017; 11: S53-56.

18. Tandon N, Kalra S, Balhara Y P S, Baruah M P, Chada M P, Chandalia M P et al. Forum for injection technique (FIT), India: The Indian recommendations 2.0, for best practice in insulin injection technique, 2015. Indian Journal of Endocrinology and Metabolism. 2015; 19: 317-31.

19. Johansson U B, Amsberg S, Hannerz L, Wredling R, Adamson U, Arnqvist $\mathrm{H} \mathrm{J}$ et al. Impaired absorption of insulin as part from liopohypertrophy injection sites. Diabetes Care. 2005; 28: 2025-27.

20. Danish Nurses Organization. Evidencebased Clinical Guidelines for Insulin for 
Adults with Diabetes Mellitus, $2^{\text {nd }}$ edn. DNO. Copenmark, 2006.

21. Association for Diabetes care Professionals (EADV). Guideline: The Administration of Insulin with the Insulin Pen. EADV, Utrecht, Netherlands, 2008.

22. Jehle PM, Pens: Richtige Mischung = weniger Unterzuckerunger, Diabetes J. 2000; 5: 46-50.

23. Muller N, Frank T, Kloos C, Lehmann T, Wolf G, Muller UA. Randomized crossover study to examine the necessity of an injection-to-meal interval in patients with type 2 diabetes and human insulin. Diabetes Care. 2013; 36:1865-69. 